Musées, Patrimoine et Culture scientifiques et techniques

112 | 2007

juillet - août 2007

\title{
Expositions permanentes : grands récits ou fragments de discours
}

\section{Dominique Botbol}

\section{(Q) OpenEdition \\ Journals}

Édition électronique

URL : http://journals.openedition.org/ocim/727

DOl : $10.4000 /$ ocim.727

ISSN : 2108-646X

Éditeur

OCIM

Édition imprimée

Date de publication : 1 juillet 2007

Pagination : 4-11

ISSN : 0994-1908

Référence électronique

Dominique Botbol, «Expositions permanentes : grands récits ou fragments de discours », La Lettre de I'OCIM [En ligne], 112 | 2007, mis en ligne le 15 février 2011, consulté le 01 mai 2019. URL : http:// journals.openedition.org/ocim/727 ; DOI : 10.4000/ocim.727 
en ont été les organisations et quels ont été les principaux souhaits d'amélioration exprimés par les visiteurs ? Il était indispensable de tirer les enseignements du passé avant de construire la troisième génération d'« Explora ».

\section{Les deux premières générations d'" Explora"}

La première génération, de 1986 à 1991

« Explora » est conçu sur la base de deux principes alors novateurs, une organisation thématique transdisciplinaire et une muséologie interactive. « Explora » comporte alors 18 expositions permanentes regroupées en 4 secteurs thématiques. Les quatre secteurs affichent une ambition encyclopédique :

- secteur 1 De la Terre à l'Univers,

- secteur 2 L'aventure de la vie,

- secteur 3 Matière et travail de l'homme,

- secteur 4 Langages et communication.

Les secteurs sont implantés sur trois tranches transversales sur trois niveaux (secteurs 1, 2, 3) et une tranche longitudinale du bâtiment (secteur 4).

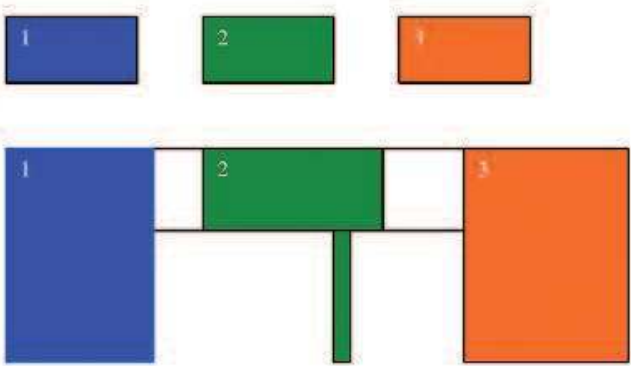

Explora niveau 2 et 3

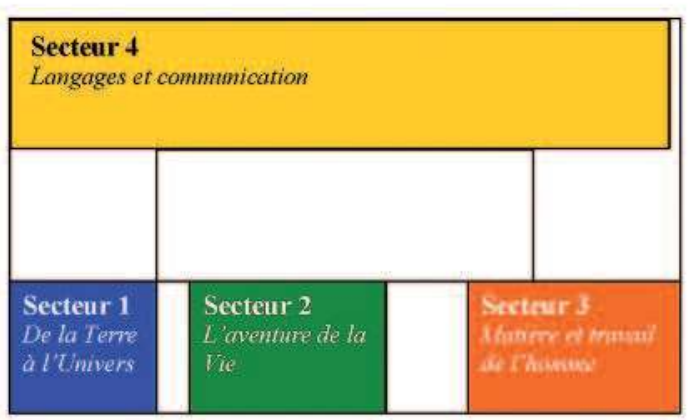

Explora niveau 1

1986 à 1991, première génération d'« Explora » (-) CSI/D. Botbol

Dans ce dispositif, il apparaît très vite qu'à l'exception notable du secteur Langages et communication qui se développe longitudinalement sur un seul niveau au nord du bâtiment, les visiteurs ne repèrent pas ces secteurs car ils ne correspondent pas à leur pratique de visite par niveau ; ils ne perçoivent donc pas l'organisation d'« Explora » en macro unités thématiques. Ils se retrouvent dès lors face à une liste non hiérarchisée de 18 thèmes - et l'on sait qu'il est très difficile de mémoriser plus de 4 à 5 items dans une liste.

"La cohérence spatiale implique souvent l'absence d'une logique thématique; un choix (de visite) porté sur la cohérence des contenus implique des itinéraires dépourvus de continuité...

La discontinuité de l'espace et des contenus entraîne la perception d'un espace éclaté. Cette perception est renforcée par la multiplication des interconnexions et les interpénétrations entre les différents îlots, qui renforcent la perception d'une continuité spatiale sans relation avec les contenus et les objets exposés » (2).

La plupart des expositions traitent de sciences dures ou de technologie avec deux exceptions notables : Expressions et comportements qui relève de la psychologie comportementale et de l'anthropologie (rituels universaux) et Images qui comporte une dimension de critique sociale. Ces deux expositions figurent parmi les expositions les plus largement appréciées par les visiteurs.

La muséographie initiale est inspirée pour une grande part de la muséologie " hands-on » des anglosaxons : les expositions proposent un grand nombre de manips physiques interactives typiques des musées des sciences, chaque manip délivrant un fragment de discours ; il n'y a pas de frontière très marquée entre chaque exposition et les maîtres mots sont liberté et interactivité.

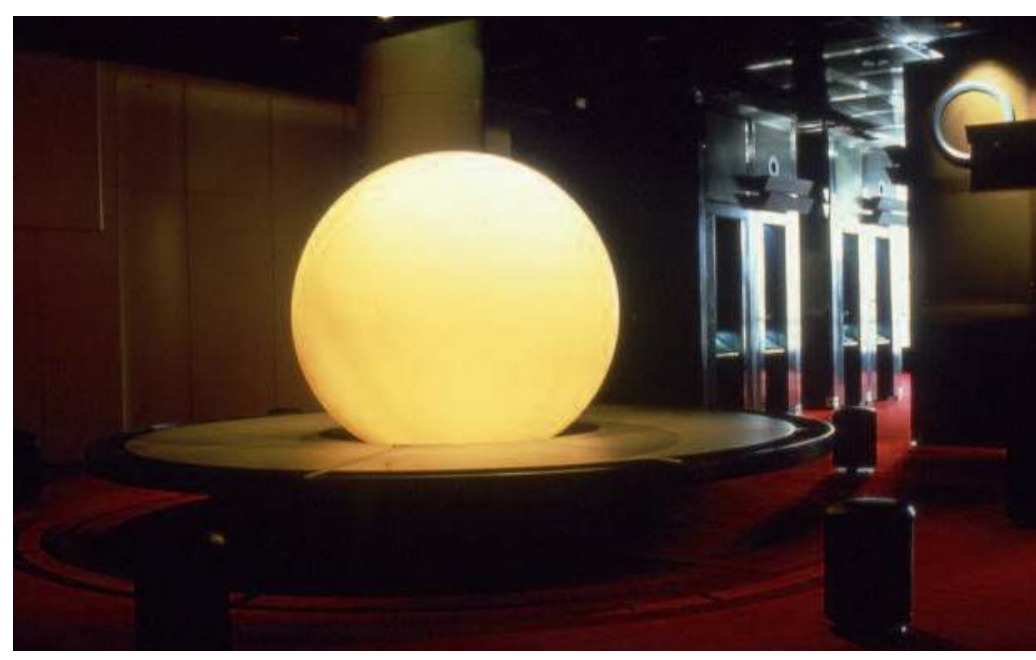

1986, exposition permanente Le Monde sonore (c) CSI/P. Astier-Blue Line 
La deuxième génération, de 1992 à 2001

La Cité des Sciences et de l'Industrie apprivoise son bâtiment et connaît mieux ses publics. Elle entreprend de renouveler ses expositions permanentes en faisant évoluer la muséographie initiale principalement sur trois points.

1/ Puisque les études de public établissent que les 4 secteurs thématiques transversaux ne correspondent pas à la perception et à la pratique des visiteurs, il est décidé de leur préférer une description d'« Explora » en 3 unités géographiques horizontales beaucoup plus logiques avec la déambulation naturelle des visiteurs et correspondant tant bien que mal à des unités de sens. Paru en 1996, le Grand guide du musée Explora décrit ainsi l'organisation des expositions permanentes : " La galerie sud propose une déambulation à travers six pôles d'activité et de réflexion de la société industrielle contemporaine. La galerie nord est consacrée aux outils sensoriels, conceptuels et techniques qui permettent à l'homme d'appréhender le monde et de le communiquer. Le balcon nord et les mezzanines mettent en scène quelques-unes des questions fondamentales que se pose l'homme sur lui-même, sur la Terre et sur l'Univers".
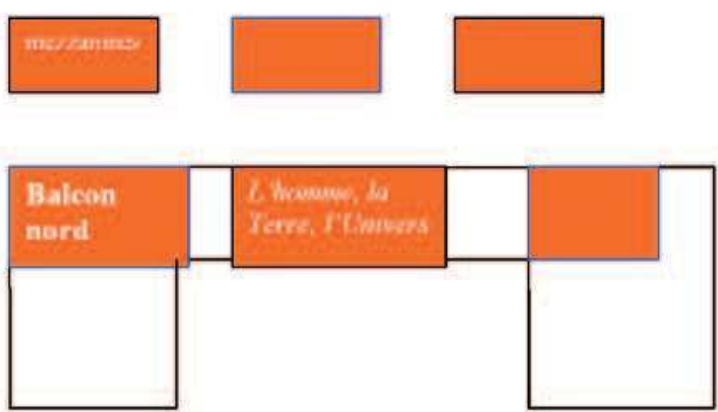

Explora niveau 2 et 3

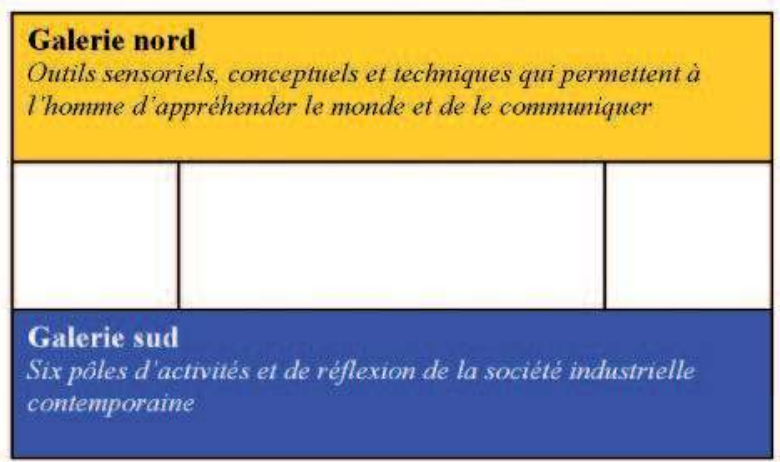

Explora niveau 1

1992 à 2001, deuxième génération d'« Explora » (c) CSI/D. Botbol
2/ La Cité réinterroge ses missions, elle balance entre le centre de sciences et le musée de civilisation. Elle s'affirme comme le lieu où le grand public découvre ce que font ensemble les sciences et l'industrie et où le visiteur citoyen peut réfléchir sur leur impact sur la vie quotidienne et sur les sociétés, se démarquant ainsi du Palais de la Découverte dédié aux sciences fondamentales. Les contenus des expositions sont ainsi plus en prise avec la réalité de la recherche et des applications industrielles. Ainsi, la seconde génération de l'exposition Les Sons bénéficie de nombreux partenariats industriels et montre toute une série d'applications industrielles, alors que la version initiale était essentiellement tournée vers la physique et la perception. Avec L'Homme et la santé en 1993, puis L'Homme et les gènes en 2002, les questions de société et d'éthique prennent leur place dans les expositions permanentes. Avec la création de " Science Actualités », une infrastructure permanente permet de traiter des questions d'actualité scientifique. Les études de public font ressortir que " ce qui motive la curiosité et le désir de comprendre, c'est l'actualité de la thématique traitée: les visiteurs parlent spontanément de sujets de société, de problèmes d'actualité » (3). "À propos des OGM par exemple, on ne sait pas trop quelle position prendre, mais là, on a l'impression qu'on peut avoir un avis làdessus » (4).

3/ À l'intérieur des grandes entités thématiques, les expositions se referment sur elles-mêmes et proposent un parcours plus explicite. On l'a vu, les études ont montré que le tissu fragmenté et banalisé de manips interactives dans la succession des différents thèmes des secteurs initiaux a été perçu par les visiteurs

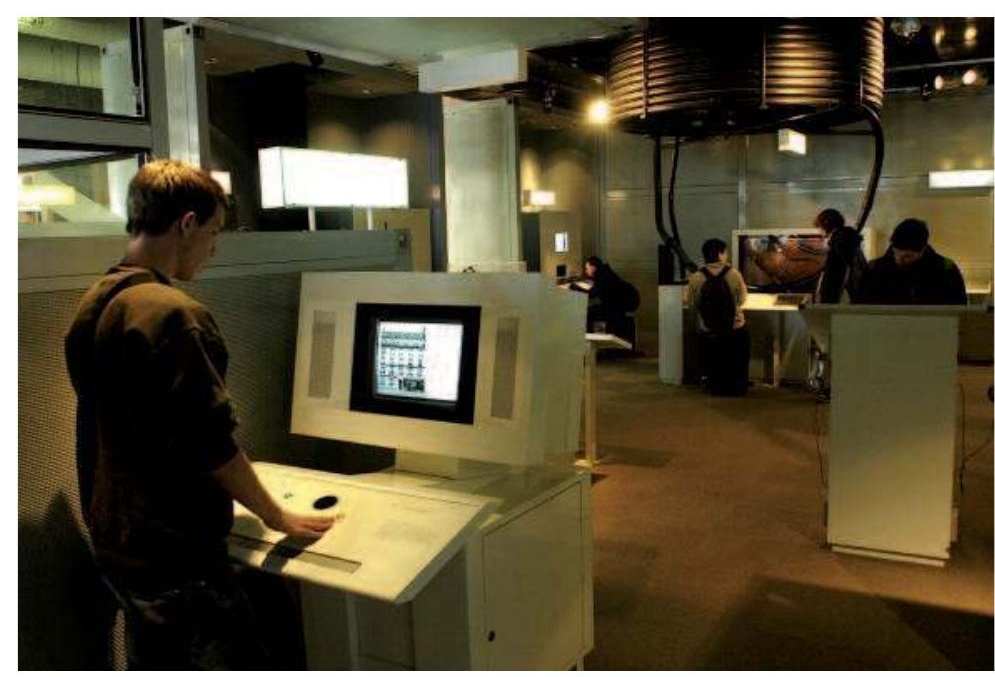

1999, exposition permanente Les Sons (C) CSI/P. Astier-Blue Line 
comme source de confusion et de désorientation. Chaque nouvelle exposition est donc munie d'une entrée principale et d'une enceinte. Un couloir de circulation est ménagé entre les expositions.

Ainsi, l'exposition L'Homme et les gènes est constituée d'un parcours en 4 grandes salles, chacune consacrée à une thématique :

- Le vivant et l'évolution (comment la reproduction crée la diversité et permet l'évolution du vivant),

- La part des gènes (quelle est la part des gènes dans notre identité d'humains),

- Le génie génétique (les nouveaux pouvoirs de manipulation du vivant),

- Questions de société (les débats scientifiques, les enjeux éthiques et philosophiques).

Les appréciations des visiteurs recueillies par l'Observatoire des publics montrent que les visiteurs saluent la clarté de l'exposition, la simplicité du parcours et l'approche pédagogique ${ }^{(5)}$.

"C'est très varié, soit on s'assoit, soit on écoute, il y a des lumières... un cheminement qui nous guide, donc il y a un sens déjà. On ne se balade pas n'importe comment, on a l'impression d'être vraiment guidés... » "Très bien présentée; fluide; parcours indiqué à l'entrée; bien documentée...»

"Bien faite et amusante, on y apprend beaucoup de choses; bien faite et agréable à parcourir : il y a un parcours bien fait scandé d'écrans intéressants ».

Mais, si la lisibilité de la topographie des lieux s'améliore sensiblement à l'intérieur des nouvelles expositions, le problème d'un sens de visite plus clair n'est pas résolu à l'échelle d' « Explora » dans son ensemble, comme le montre la présence récurrente en première position du souhait d'un "sens de visite plus clair » dans les enquêtes de l'Observatoire des publics conduites tout au long de ces années. Voici d'après une liste de 20 souhaits d'amélioration les 4 souhaits d'amélioration choisis en priorité par les visiteurs interrogés entre 1997 et 2004 :

- en 1997 : un sens de visite plus clair (24\%), une présentation générale du musée « Explora » (19,5\%), plus de professionnels pour répondre aux questions (14\%), moins de pannes (11\%);

- en 1999 : plus de professionnels pour répondre aux questions (31\%), un sens de visite plus clair (30\%), moins de pannes $(27 \%)$, une présentation générale du musée « Explora » $(23 \%)$;

- en 2000 : un sens de visite plus clair (26\%), moins de pannes $(26 \%)$, plus de professionnels dans les expositions $(24 \%)$, une présentation générale du musée «Explora » $(17 \%)$;

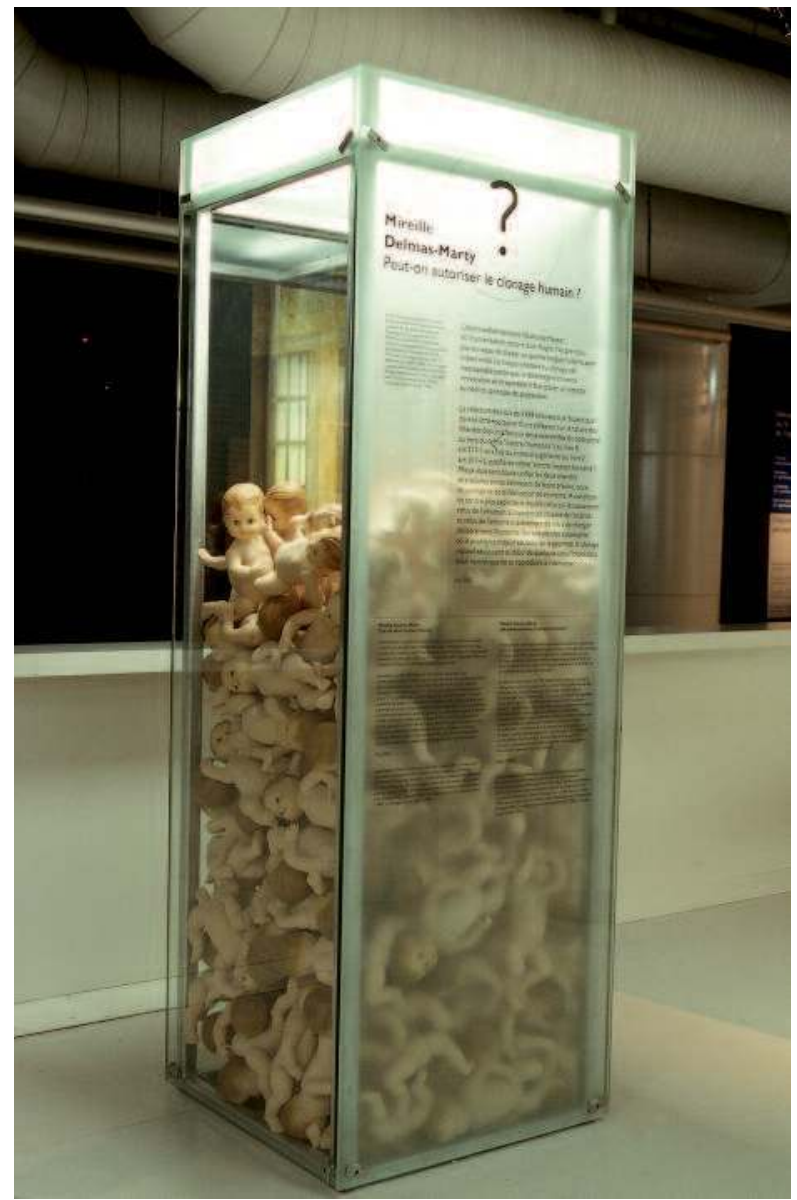

2002, exposition permanente L'Homme et les gènes: questions de société
(c) CSI/P. Astier-Blue Line

- en 2002 : un sens de visite plus clair (30\%), plus de professionnels pour répondre aux questions (28\%), moins de pannes $(21 \%)$, une présentation générale du musée «Explora » $(13 \%)$;

- en 2004 : un sens de visite plus clair (32\%), plus de professionnels pour répondre aux questions (29\%), moins de pannes (27\%), moins de monde devant les interactifs (20\%), une présentation générale du musée « Explora » $(17 \%)$.

Les études montrent cependant qu'il ne s'agit pas seulement d'un problème d'orientation, notamment depuis la pose en 1998 de grandes bâches de signalétique qui forment un sommaire géant de l'offre des expositions, et qui a eu pour effet immédiat de faire reculer en $4^{\mathrm{e}}$ position, à partir de 1999 , la demande d'une "présentation générale du musée Explora». Il semble que les visiteurs expriment ainsi plutôt le souhait de "suivre un sens de visite à l'intérieur d'une exposition et de comprendre le lien entre les expositions et le sens général d'Explora ». Citons quelques remarques de visiteurs, recueillies en 2002 (6) : 
Pour répondre à ces objectifs, nous avons élaboré une proposition fondée sur deux axes, accompagnée d'une volonté permanente de renouvellement et de diversification des formes muséographiques :

- des expositions de référence regroupées en trois grands récits, au nord, sur environ $6000 \mathrm{~m}^{2}$;

- une galerie de l'innovation, au sud, sur environ $4000 \mathrm{~m}^{2}$.
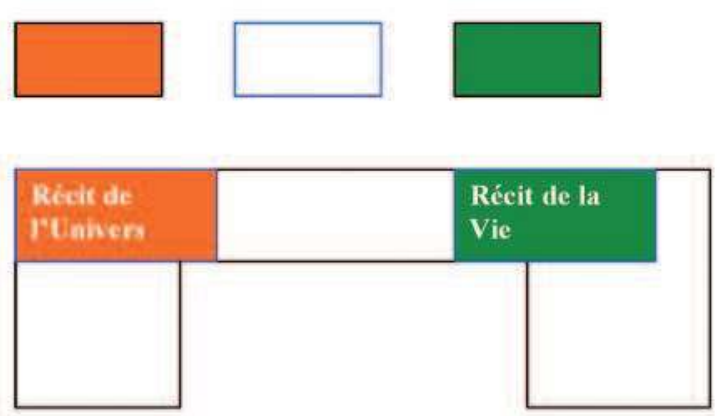

Explora niveau 2 et 3

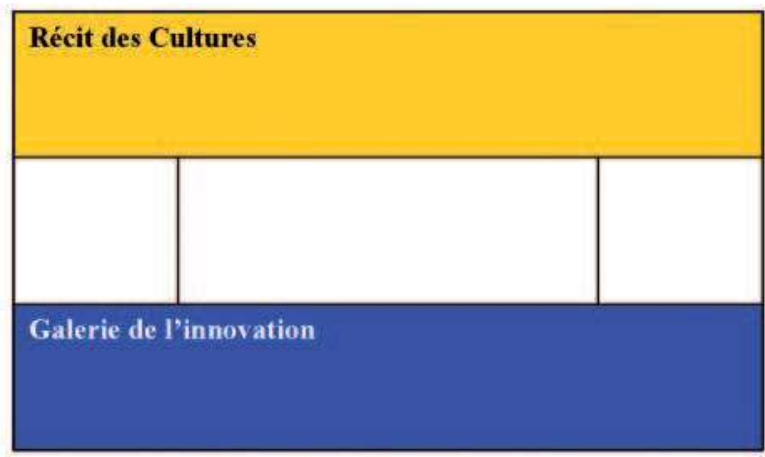

Explora niveau 1

2006 à 2012, troisième génération d'« Explora » en projet ๑) CSI/D. Botbol

À la suite d'une séance du séminaire de muséologie du 20 janvier 2006 intitulé Expositions permanentes : grands récits ou fragments de discours, la réflexion se poursuit dans le cadre d'un atelier interne à la Cité des Sciences et de l'Industrie ${ }^{(9)}$.

En outre, des conventions ont été établies par le département Évaluation et Prospective de la Cité des Sciences et de l'Industrie avec des laboratoires de recherche du CNRS, notamment le CERLIS, pour procéder à l'évaluation des différents projets de rénovation des expositions permanentes actuellement en cours, notamment Le grand récit de l'univers et La galerie de l'innovation (10).
Nous proposons dans ce qui suit de faire part des réflexions en cours concernant plus particulièrement le projet des trois grands récits.

\section{Les 3 grands récits}

«Savoir, c'est se souvenir » : pour organiser les expositions de référence, nous avons choisi de nous inspirer de la pensée de Michel Serres exposée dans L'Incandescent (11). Cet essai philosophique, paru aux Éditions Le Pommier en 2003, montre que le grand enseignement des sciences modernes est que tout dans l'Univers a une histoire et se définit par son histoire, y compris ce qui paraissait stable et immuable comme la matière. Il fait valoir que chaque être humain porte en lui trois mémoires emboîtées :

- la mémoire culturelle, la plus récente, depuis l'hominisation ;

- la mémoire biologique : découverte au cours du dernier demi-siècle, l'universalité du code génétique réunit les hommes entre eux et jusqu'aux bactéries les plus élémentaires ; tout vivant porte en lui la trace de naissance de la vie, voilà plus de trois milliards d'années ;

- la mémoire de l'univers, car certains des atomes qui nous constituent ont été créés au moment du big bang.

Les expositions permanentes de la Cité des Sciences et de l'Industrie raconteront cette histoire à travers trois grands récits aux temporalités emboîtées :

- Le grand récit de l'univers : le premier volet présentera, sous la forme d'une vaste enquête, les origines de la matière il y a 15 milliards d'années et son histoire. Les objets de l'univers - la matière, la lumière, l'énergie - seront les héros de la découverte. Tandis que dans le second volet, consacré aux lois physiques de l'univers, le visiteur deviendra le héros de la démarche, et aura notamment l'occasion de retrouver les grandes figures liées aux découvertes des lois physiques et cosmologiques, de Newton à Einstein.

- Le grand récit de la vie : présentera les connaissances scientifiques les plus récentes en biologie, génétique et médecine, mises en perspective par rapport à la longue histoire de l'évolution du vivant, depuis l'apparition de la vie sur terre avec la première bactérie il y 3,4 milliards d'années jusqu'à l'homme contemporain. - Le grand récit des cultures : racontera, depuis l'apparition de l'homo sapiens, il y a 100000 ans, l'histoire des innovations fondatrices accomplies par l'espèce humaine pour comprendre, représenter, communiquer et penser le monde. Le visiteur pourra ainsi 


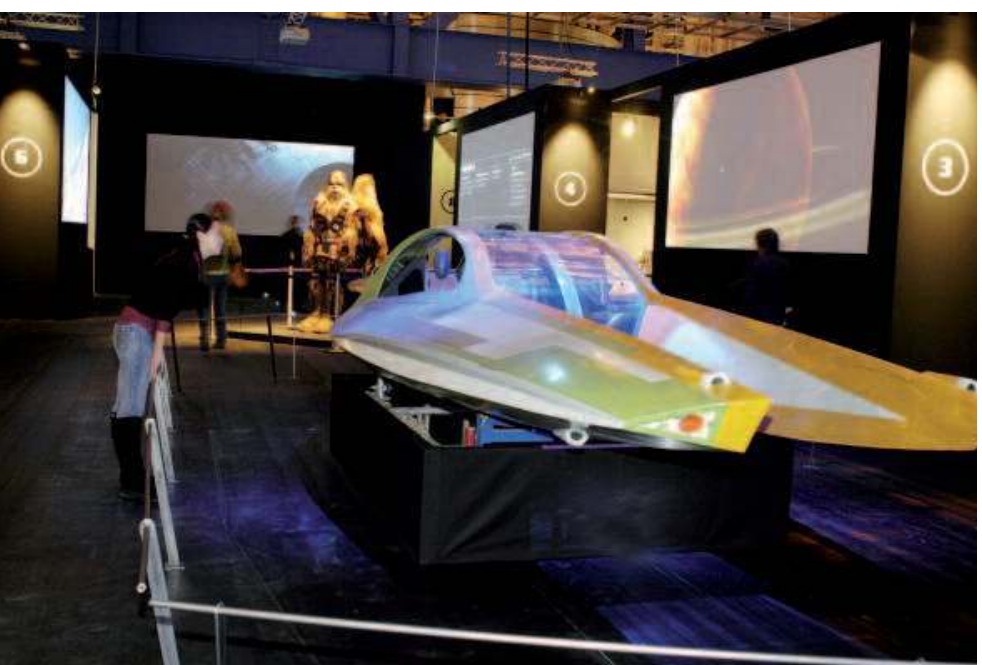

2005-2006, exposition temporaire Star Wars, I'expo (๖) CSI/B. Baudin-Le Bar Floréal

déterministe quand on se retourne. Comme tous les récits, celui-ci, le plus grand et le plus véridique de tous, déploie le temps contingent du chaos »(13).

Ainsi, dans un double mouvement paradoxal, nous allons tenter de donner à nos visiteurs des repères rassurants et stables et en même temps introduire une distanciation critique en montrant comment le savoir scientifique est en perpétuel reconstruction : le récit de l'univers diffère quand il est raconté par Newton ou par Einstein. Ce faisant, nous ne ferons que suivre les recommandations du grand dramaturge Bertold Brecht, qui nous dit qu'il ne peut y avoir de distanciation s'il n'y a pas d'identification. Autrement dit, pour donner accès à la complexité du monde, il faut ménager pour nos visiteurs un temps d'appropriation avant la mise en distanciation critique.

\section{Notes}

(1) L'Observatoire des Publics a été créé dès l'ouverture de la Cité des Sciences et de l'Industrie par le département Évaluation et Prospective (DEP). Ce département est actuellement placé sous la responsabilité de Avmard de Mengin.

(2) «Explora ", orientation spatiale et conceptuelle dans le contexte de la première visite, analyse des attentes, synthèse. Département Évaluation et Prospective, juin 1993, étude réalisée avec la collaboration de Bernadette Grossir-Le Nouvel, François Mounier et Walter Detomasi de la société AREA.

(3) Les visiteurs, synthèse des études 1986-2004. Aymard de Mengin, Marie-Claire Habib, DEP 2005, portraits de visiteurs, L'Homme et les gènes, p. 40.

(4) Défis du vivant : portraits de visiteurs. Virginie Loisel, Marie-Claire Habib, Agnès Suillerot, CSI, DEP 2004.

(5) L'Homme et les gènes : synthèse des données de l'observatoire des publics. Pierre Cohen-Hadria, CSI, DEP 2003.
(6) et (7) Aymard de Mengin, note intitulée Les visiteurs d'« Explora » en 2002, datée du 15 janvier 2003.

(8) Ces ateliers étaient organisés dans le cadre du projet de rénovation global de la Cité des Sciences et de l'Industrie. Les réflexions de l'atelier consacré aux expositions permanentes ont abouties aux deux documents suivants : Les expositions durables, Réal Jantzen, octobre 2003 et Contribution au projet de rénovation, Roland Schaer, septembre 2003.

(9) Cet atelier, animé par Anne Stephan et Jean-Paul Natali (CSI), rassemble des muséographes de la Cité des Sciences et de l'Industrie et des experts invités comme Yves Jeanneret, professeur en Sciences de l'Information et de la Communication (CELSA-Paris IV), Magali Roux, directeur de recherche CNRS Institut Pasteur.

(10) Jacqueline Eidelman et ses équipes réalisent des enquêtes et réunissent des groupes témoins pour les associer à un processus d'évaluation, d'élaboration et de réflexion sur le choix des thèmes et les modes de traitement des expositions dont le renouvellement est actuellement en projet.

(11) Michel Serres est professeur à Stanford University, membre de l'Académie française ; il est l'auteur de très nombreux essais philosophiques et d'histoire des sciences.

(12) Regards croisés sur neuf expositions permanentes d' Explora", Jacqueline Eidelman (Cerlis Paris-V), Daniel Jacobi (université d'Avignon), Annette Viel (université de Bourgogne), Marie-Claire Habib, CSI, DEP 2001.

(13) Serres, M. L'Incandescent, Paris : Éditions Le Pommier, 2003, p. 26.

\section{Bibliographie}

Serres, M. L'Incandescent. Paris : Éditions Le Pommier, 2003.

Observatoire des Publics, département Évaluation et Prospective de la Cité des Sciences et de l'Industrie (Aymard de Mengin, Marie-Claire Habib, Agnès Suillerot).

"Explora », orientation spatiale et conceptuelle dans le contexte de la première visite, analyse des attentes, synthèse, département Évaluation et Prospective, juin 1993, étude réalisée avec la collaboration de Bernadette Grossir-Le Nouvel, François Mounier et Walter Detomasi de la société AREA.

Les visiteurs, synthèse des études 1986-2004, Aymard de Mengin, MarieClaire Habib, CSI, DEP, 2005

Défis du vivant : portraits de visiteurs, Virginie Loisel, Marie-Claire Habib, Agnès Suillerot, CSI, DEP, 2004.

L'Homme et les gènes : synthèse des données de l'observatoire des publics, Pierre Cohen-Hadria, CSI, DEP, 2003.

Les visiteurs d'« Explora » en 2002, Aymard de Mengin, note interne datée du 15 janvier 2003.

Regards croisés sur neuf expositions permanentes d' "Explora ", département Évaluation et Prospective de la Cité des Sciences et de l'Industrie, Jacqueline Eidelman (Cerlis Paris-V), Daniel Jacobi (université d'Avignon), Annette Viel (université de Bourgogne), Marie-Claire Habib, 2001

Les expositions durables, Réal Jantzen, chargé de mission, document interne de la Cité des Sciences et de l'Industrie, octobre 2003

Contribution au projet de rénovation de la Cité des Sciences et de l'Industrie, Roland Schaer, directeur Sciences et Société, document interne de la Cité des Sciences et de l'Industrie, septembre 2003. 